\title{
OPTICAL ABSORPTION BY INCOHERENT AND COHERENT ENSEMBLES OF SPIN EXCITATIONS IN AN UNPOLARISED QUANTUM HALL SYSTEM
}

\author{
S. Dickmann \\ Institute of Solid State Physics of RAS, Chernogolovka, 142432, Moscow District, Russia \\ Email: dickmann@issp.ac.ru
}

Received 25 October 2018; revised 2 April 2019; accepted 21 June 2019

\begin{abstract}
In connection with recent studies of extremely long-living spin-cyclotron excitations that are actually magnetoexcitons in the quantum Hall electron gas, we discuss the contribution to light absorption related to the presence of a magnetoexcitonic ensemble in this purely electronic system. The distribution of magnetoexcitons in an 'incoherent' phase, as well as absorption of light, is determined by a smooth random potential inevitably present in a quantum well. Since weakly interacting excitations have to obey the Bose-Einstein statistics, one may expect the appearance of a coherent phase where all magnetoexcitons are in the same state. The absorption of light is still determined by disorder in the system, but it turns out to be about an order of magnitude higher. A comparative analysis is made of both incoherent and coherent cases. The condition for coherent-incoherent phase transition is discussed. It is expected to be strongly related to long-distance inter-excitonic correlations. The latter are accounted in terms of virial correction (i.e. depending on magnetoexciton concentration) for the single magnetoexciton energy found within the approximation of a slightly non-ideal gas.
\end{abstract}

Keywords: two-dimensional electron gas, quantum well, magnetic field, optical absorption, resonant Rayleigh reflection, magnetoexciton, cyclotron spin-flip excitation, Bose-Einstein condensation

PACS: 73.21.Fg, 73.43.Lp, 78.67.De

\section{Introduction (cyclotron spin-flip excitation)}

Cyclotron spin-flip exciton (CSFE) in the $v=2$ quantum Hall system is the lowest-energy excitation [13] and has an extremely long lifetime. The latter is theoretically estimated to be up to several milliseconds [4 5]. As is usually the case in relaxation problems, the time actually turns out to be shorter due to the presence of additional relaxation channels which could hardly be predicted before a specific experimental study. In fact, the CSFE relaxation found experimentally in the unpolarised quantum Hall system created in a GaAs/AlGaAs heterostructure reaches $100 \mu \mathrm{s}$ [ 6 ] that seems to be a record value for a delocalised state excited in the conduction-band electron system. Such a slow relaxation suggests that the excitation ensemble obeying the Bose-Einstein statistics can, at a sufficiently high concentration, experience a transition to a coherent state, i.e. BoseEinstein condensate. Note that both CSFE creation and CSFE monitoring are performed by optical methods [3, 6]. In this respect, it is of interest to study the contribution to light absorption related to the CSFE ensemble in the 2DEG. In the present work we perform a comparative analysis of absorption by the CSFE ensemble in incoherent and coherent phases. (This also strongly correlates with light emission if the resonant reflection technique is used [6].)

The CSFE is a solution of the many-electron Schrödinger equation with a $\delta S=1$ change of 
the total spin as compared to the ground state where $S=0$. In other words, generally, this excitation is a triplet with $S=1$ and $S_{z}=1,0,-1$. All the three components have equidistant energies gapped by the Zeeman value $\left|g \mu_{\mathrm{B}} B\right|$. The lowest-energy component corresponds to $S_{z}=1$ because the $g$-factor is negative in $\mathrm{GaAs}$ heterostructures. In the present study we consider only such $S=S_{z}=1$ magnetoexcitons. A noticeable concentration of such excitations, $N / \mathcal{N}_{\phi} \lesssim 0.1\left(\mathcal{N}_{\phi}\right.$ is the total number of states in the Landau level), can be achieved experimentally [6]. At high concentrations the inter-excitonic (CSFE-CSFE) interaction seems to become fairly strong. Yet, in the following we study an exciton ensemble only in the 'dilute limit', thus ignoring CSFECSFE coupling. Due to the very long CSFE relaxation time we consider an exciton ensemble as a metastable system with a given number of excitons $N$.

The dependence of CSFE energy on the 2D momentum $\mathbf{q}$ in the $v=2$ unpolarised quantum Hall system was first calculated by Kallin and Halperin [1]. The authors studied the problem within the first order in a small parameter given by the ratio

$$
r_{\mathrm{s}}=E_{\mathrm{C}} / \hbar \omega_{\mathrm{c}}^{(e)}
$$

of the characteristic Coulomb energy $E_{\mathrm{C}}$ to the cyclotron energy $\hbar \omega_{c}^{(e)}\left(E_{C} \lesssim e^{2} / \kappa l_{B}\right.$, where $\kappa$ is the dielectric constant, $l_{B}=\sqrt{c \hbar / e B}$ is the magnetic length). Besides, they considered the ultra two-dimensional limit in the absence of any disorder. Indeed, the CSFE energy counted off the ground-state level is determined by the formula $E_{\mathbf{q}}=\delta^{(0)}+\mathcal{E}_{q}$, where

$$
\mathcal{E}_{q}=\int_{0}^{\infty} \mathrm{d} s \mathrm{e}^{-s^{2} / 2} \mathcal{F}_{e e}(s)\left(1-\frac{s^{2}}{2}\right)\left[1-J_{0}(s q)\right]
$$

is the $q$-dispersion (here and everywhere below momentum $\mathbf{q}$ is measured in $1 / l_{B}$ units; $J_{0}$ is the Bessel function), and

$$
\delta^{(0)} \equiv \hbar \omega_{\mathrm{c}}^{(e)}-\left|g \mu_{\mathrm{B}} B\right|+\varepsilon_{0}
$$

is the $q=0$ energy including cyclotron and Zeeman energies, and the negative Coulomb shift $\varepsilon_{0}$ remaining nonzero even if $q \rightarrow 0$. (The $\varepsilon_{0}<0$ value representing the second-order Coulomb correction $\left(\sim \hbar \omega_{c}^{(e)} r_{s}^{2}\right)$ calculated in the work [2] and experimentally measured in [3].) The form factor is

$$
\mathcal{F}_{e e}(q)=\frac{e^{2}}{\kappa l_{B}} \iint \mathrm{d} z_{1} \mathrm{~d} z_{2} \mathrm{e}^{-q\left|z_{1}-z_{2}\right| l_{B}}\left|\chi_{e}\left(z_{1}\right) \chi_{e}\left(z_{2}\right)\right|^{2},
$$

where $\chi_{e}(z)$ describes the electron size-quantised functions in the quantum well. (The $\chi_{e}(z)$ function in a quantum Hall system should be $B$-dependent.) The CSFE $q$-dispersion for the $v=1$ filling is the same as $\mathcal{E}_{q}$ in the $v=2$ case if obtained within the 'single-mode approximation'. So, an example of the calculation (2) for a certain real system is presented, e.g. in Ref. [帄. It shows a very weak $q$-dispersion: $\left|\mathcal{E}_{q}\right| \lesssim 0.01 E_{\mathrm{C}}$ down to $q \sim 1\left(\sim 1 / l_{B}\right.$ in common units). It is worth noting that there are various ways to compute the form factor (3) based on different calculation/modelling of the function $\chi_{e}(z)$. Approaches were discussed earlier in the review [8] (see also Refs. [2, 4, 5, ,7). However, practice shows that in a quantum Hall system (in a strong magnetic field) a simple semi-empirical formula

$$
\mathcal{F}_{e e}(q) \simeq \frac{e^{2}}{\kappa l_{B}(1+d q)}
$$

works quite well for wave vectors $q \lesssim 1$, where the fitting parameter $d$, proportional to the effective width of the quantum heterostructure, is measured in $l_{B}$ units.

In the absence of any interaction mixing spatial and spin variables, the Hamiltonian of a translationally invariant quantum Hall system has the following form:

$$
\hat{H}=-\left|q \mu_{\mathrm{B}} B\right| \hat{S}_{z}+\hat{H}^{(1)}+\hat{H}_{\text {Coul }} .
$$

Here $\hat{S}_{z}$ is the $z$ component of the total spin operator of electrons, $\hat{H}^{(1)}$ is the 'kinetic energy' operator of electrons and $\hat{H}_{\text {Coul }}$ is the Coulomb interaction operator. We recall the eigenfunctions of the operator $\hat{H}^{(1)}$

$$
\psi_{n p}(\boldsymbol{r}) \propto H_{n}\left(\frac{x-x_{p}}{l_{B}}\right) \exp \left[-\frac{\left(x-x_{p}\right)^{2}+2 \mathrm{i} x_{p} y}{2 l_{B}^{2}}\right],
$$

representing the $p$-orbital function of the $n$th Landau level. (The Landau gauge is used: $H_{n}$ is the Hermite polynomial, $x_{p}=-p l_{B}, p$-numbers are measured in $1 / l_{B}$ units.)

Using the 'excitonic representation' technique (see the Appendix below) the CSFE with the 2D momentum $\mathbf{q}$ is presented in the form of action of 
the exciton creation operator $\mathcal{Q}_{\mathrm{q}}^{\dagger}$ on the ground state $|0\rangle$. Specifically,

$$
Q_{\mathbf{q}}^{\dagger}=N_{\phi}^{-1 / 2} \sum_{p} \mathrm{e}^{-\mathrm{i} q_{x}\left(p-q_{y} / 2\right)} b_{p}^{\dagger} a_{p-q_{y}}
$$

( $\mathcal{Q}$-operators as applied to a quantum Hall system were first used in works [10, 11]). In our case $|0\rangle$ denotes the $v=2$ ground state with a fully occupied zero Landau level; $a_{p}$ is the operator annihilating an electron on the upper spin sublevel of the zero Landau level with spin down, i.e. antiparallel to the magnetic field, and $b_{p}^{\dagger}$ creates an electron on the first Landau level with the spin directed along the magnetic field. The operators in Eq. (5) can be expressed in terms of the excitonic representation [see Eqs. (A3) and (A5) in the Appendix]. The commutators with $\mathcal{Q}_{\mathrm{q}}^{\dagger}$ satisfy the following properties: $\left[\hat{S}_{z}, \mathcal{Q}_{\mathbf{q}}^{\dagger}\right]=-1,\left[\hat{H}^{(1)}\right.$, $\left.\mathcal{Q}_{\mathbf{q}}^{\dagger}\right]=\hbar \omega_{c}$. In the following, considering the Coulomb interaction, we will limit ourselves only to the first order in $r_{s}$. This means that in the $\hat{H}_{\text {Coul }}$ operator one may keep only the terms commuting with $\hat{H}^{(1)}$, thus preserving the cyclotron energy of the studied states, and certainly preserving the spin state due to the well-known property of the Coulomb interaction. These terms can be extracted from a general expression of $\hat{H}_{\text {Coul }}$. Designating them as $\hat{H}_{\text {Coul }}^{\prime}$ (see the Appendix) we have

$$
\left[\hat{H}_{\text {Coul' }}^{\prime} \mathcal{Q}_{\mathbf{q}}^{\dagger}\right]|0\rangle=\mathcal{E}_{q} \mathcal{Q}_{\mathbf{q}}^{\dagger}|0\rangle
$$

The CSFE represents a purely electronic kind of magnetoexciton [9] where the quantum mechanical average of the distance between the positions of a promoted electron and an effective 'hole' (a vacancy in the spin-down sublevel of the zero Landau level) is equal to $\Delta \mathbf{r}=l_{B} \mathbf{q} \times \hat{z}$ [1]. Therefore, this excitation possesses an electric dipole-momentum

$$
\mathbf{d}_{\mathbf{q}}=e l_{B} \mathbf{q} \times \hat{z} .
$$

\section{Light absorption by an incoherent excitonic ensemble}

First, we study the incoherent state of the CSFE ensemble

$$
|N\rangle=\mathcal{Q}_{\mathbf{q}_{N}}^{\dagger} \mathcal{Q}_{\mathbf{q}_{N-1}}^{\dagger} \ldots \mathcal{Q}_{\mathbf{q}_{1}}^{\dagger}|0\rangle,
$$

considering the general case under the condition $N \ll \mathcal{N}_{\phi}$ where all qs are assumed to be different.
One can find that the squared norm has the following property:

$$
\langle N \mid N\rangle=\left[1+O\left(N / \mathcal{N}_{\phi}\right)\right]\langle N-1 \mid N-1\rangle .
$$

The perturbation operator responsible for light absorption has the form

$$
\hat{\mathcal{P}}=A \sum_{p} V_{p}^{\dagger} a_{p}^{\dagger},
$$

where $V_{p}^{\dagger}$ is the creation operator of a valence heavy hole, and $A$ is a certain constant. The operator (12) is uniquely determined by two features of absorption: (i) in this case only 'vertical' electronic transitions are relevant, i.e. the photon generates a pair of a valence hole and an $a$-sublevel electron, both in the same intrinsic $p$-states of their Landau levels; (ii) all the $p$-states equiprobably participate in the absorption process. Such properties of the light absorption are related to the condition $\mathcal{L} k_{\text {photon\| }} \ll 1$ where the length $\mathcal{L}$ is the characteristic of electron 2D-density spatial fluctuations and $k_{\text {photon\|l }}$ is the photon wave vector component parallel to the electron system plane. This condition is actually satisfied [6]. The action of the $\hat{\mathcal{P}}$ operator on the state $|N\rangle$ results in the $A \Sigma_{i}\left|\mathrm{f}, \mathbf{q}_{\mathrm{i}}\right\rangle$ combination of $N$ states:

$$
\left|\mathrm{f}, \mathbf{q}_{i}\right\rangle=-\hat{\chi}_{\mathbf{q}} \prod_{j \neq i} \mathcal{Q}_{\mathbf{q}_{i}}^{\dagger}|0\rangle .
$$

Here $\hat{\chi}_{\mathbf{q}}=\mathcal{N}_{\phi}^{-1 / 2} \sum_{p} \mathrm{e}^{-\mathrm{i} q_{x}(p+q y / 2)} V_{p}^{\dagger} b_{p+q_{y}}^{\dagger}$ is the exciton operator which, by acting on the ground state, generates a valence hole and a $b$-sublevel electron. If $N \ll \mathcal{N}_{\phi}$, then neglecting any interaction of $\mathcal{Q}_{\mathbf{q}_{j}}^{\dagger}|0\rangle$ excitons with each other and with the $|v-e, \mathbf{q}\rangle=\hat{\chi}_{\mathbf{q}}^{\dagger}|0\rangle$ exciton, we find that $\left|\mathrm{f}, \mathbf{q}_{i}\right\rangle$ has a squared norm equal to $\langle N-1 \mid N-1\rangle$. With the help of Eq. (11) one obtains a matrix element squared for transition from the initial state to the final $\left|f, \mathbf{q}_{i}\right\rangle$ state. If both are appropriately normalised, it is

$$
\left|M_{i}\right|^{2}=\left\langle\left\langle\mathbf{q}_{i}, \mathrm{f}|| \hat{\mathcal{P}} \| N\right\rangle\right\rangle^{2} \approx|A|^{2}
$$

$(\langle\langle\ldots \|$ is notation for a normalized state). The following calculation of the absorption rate represents a procedure of summation over all possible final states:

$$
\mathcal{R}_{\mathrm{I}}=\frac{2 \pi}{\hbar} \sum_{i}\left|M_{i}\right|^{2} \delta\left(D_{\mathbf{q}_{i}}\right) \approx \frac{2 \pi|A|^{2}}{\hbar} \sum_{i} \delta\left(D_{\mathbf{q}_{i}}\right) .
$$


The approach used is actually single-excitonic, hence

$$
D_{\mathbf{q}}=\hbar \omega+E_{\mathbf{q}}-E_{v-e, \mathbf{q}}
$$

where $\omega$ is the probing laser-beam frequency, $E_{\mathbf{q}}$ is the total energy of the CSFE $\mathcal{Q}_{\mathrm{q}}^{\dagger}|0\rangle$, and $E_{v-e, \mathrm{q}}$ is the energy of creation of the valence-hole-conduction-electron magnetoexciton state $|v-e, \mathbf{q}\rangle$.

\subsection{Energy of the CSFE in the presence of a smooth random potential}

The following study, that is, in fact, summation of the $\delta$-functions in Eq. (15), becomes impossible without a certain concretization. This concretisation should set a specific initial state (10) representing the distribution of $\mathbf{q}_{i}$ numbers over their possible values. This distribution is established and determined by two competing effects: by thermal diffusion related to interactions with phonons, and by drift motion, where the drift velocity of magnetoexciton $\partial E_{\mathbf{q}} / \partial \mathbf{q}$ is determined by two parameters, namely, momentum $\mathbf{q}$ and smooth random electric field $\overrightarrow{\mathscr{E}}=-\nabla \varphi(\mathbf{r}), \mathbf{r}=(x, y)$. We assume that only drift motion accompanied by cooling due to phonon emission results in the establishment of an initial state (10).

First, let us study a domain with linear dimensions smaller than the spatial dispersion parameter $\Lambda$ of the smooth random potential $\varphi$ but still larger than the magnetic length. For definiteness we consider $\Lambda$ to be the correlation length of the $\varphi$ spatial distribution, the mean value of the potential being $\bar{\varphi} \equiv 0$. Within this domain we use a gradient approximation considering field $\varphi(\mathbf{R})$, as well as gradients $\nabla_{R} \varphi$ and coordinate $\mathbf{R}$ as parameters inherent to the domain (for example, $\mathbf{R}$ indicates the domain centre). So, within the domain the electrostatic term in the Hamiltonian is equal to $\hat{\varphi}=\overrightarrow{\mathscr{E}}(\mathbf{R}) \mathbf{r}$. $\mathbf{r}$ can be presented in terms of secondary quantisation: $\int \mathrm{d} \mathbf{r} \hat{\psi}^{\dagger} \mathbf{r} \hat{\psi}$, where $\hat{\psi}=\sum_{n, p}\left(\begin{array}{c}c_{n, p, \uparrow} \\ c_{n, p, \downarrow}\end{array}\right) \psi_{n, p}(\mathbf{r})$.

Then, choosing $\overrightarrow{\mathscr{E}} \| \hat{x}$, the electrostatic term takes the form

$$
\hat{\varphi}=\mathscr{E}_{x} l_{B}\left[\sum_{\sigma=\uparrow, \downarrow}\left(\hat{K}_{\sigma}^{\dagger}+\hat{K}_{\sigma}\right)-\hat{P}_{y}\right]
$$

(here $\left|\mathscr{E}_{x}\right|=|\overrightarrow{\mathscr{E}}| \equiv \mathscr{E}$ ), where

$$
\hat{P}_{y}=\sum_{n, p, \sigma=\uparrow / \downarrow} p c_{n p \sigma}^{\dagger} c_{n p \sigma}
$$

is a component of the Gor'kov-Dzyaloshinsky momentum operator $\hat{\mathbf{P}}$ rewritten in the 'generalised' form valid for purely electronic magnetoexcitons in a quantum Hall system ( $\hat{\mathbf{P}}$ is presented in $1 / l_{B}$ units). In particular, $\hat{\mathbf{P}} \mathcal{Q}_{\mathbf{q}}^{\dagger}|0\rangle=\mathbf{q} \mathcal{Q}_{\mathbf{q}}^{\dagger}|0\rangle$. $\hat{\mathbf{P}}=\Sigma \hat{\mathbf{p}}_{j}+e \mathbf{A}_{j}-e \mathbf{B} \times \mathbf{r}_{j}$ ] being a generalization of the Gor'kov-Dzyaloshinskii operator [8], plays the role of the total momentum of a magnetized 2DEG. $\left(\sum_{j}\right.$ means summation over all electrons, $\hat{\mathbf{p}}$ is the $2 \mathrm{D}$ electron momentum operator, and $\mathbf{A}$ is the $2 \mathrm{D}$ vector-potential, units where $\hbar=c=1$ are employed.) To calculate the commutator with the operator $\mathcal{Q}+$ we choose the Landau gauge, $\mathbf{A}=(0, B x)$, and, in terms of secondary quantization, obtain the following: $\hat{P}_{x}=\left(2 \pi / \mathcal{N}_{\phi}\right)^{1 / 2} \mathrm{i} \sum_{n, p, q, \sigma} \delta_{q}^{\prime}$ $c^{\dagger}{ }_{n p+q / 2 \sigma} c_{n p-q / 2 \sigma} / l_{B}$ and $\hat{P}_{y}=\Sigma_{n, p, \sigma} p c_{n p \sigma}^{\dagger} c^{\dagger}{ }_{n p \sigma} / l_{B}$, where $\delta_{x}^{\prime \prime}$ is the distributional derivative of the Dirac $\delta$-function, i.e. $\delta_{x}^{\prime}[f(x)] \equiv-f^{\prime}(0)$. The operator

$$
\hat{K}_{\sigma}^{\dagger}=\sum_{n, p} \sqrt{\frac{n+1}{2}} c_{n+1 p \sigma}^{\dagger} c_{n p \sigma}
$$

is the Landau level 'raising' operator; $c_{n p \sigma}^{\dagger}$ is the creation operator for the $n$th Landau level, for instance, $a_{p} \equiv c_{0 p \downarrow}$ and $b_{p} \equiv c_{1 p \uparrow}$. Now we obtain the contribution of the electrostatic term (17) to the magnetoexciton energy $E_{\mathbf{q}}$. The first-order correction is

$$
\begin{aligned}
& \left\langle 0\left|\mathcal{Q}_{\mathbf{q}}\right| \hat{\varphi}\left|\mathcal{Q}_{\mathbf{q}}^{\dagger}\right| 0\right\rangle \equiv-l_{B} \overrightarrow{\mathscr{E}} \times\left\langle 0\left|\mathcal{Q}_{\mathbf{q}}\right| \hat{\mathbf{P}}^{\dagger}\left|\mathcal{Q}_{\mathbf{q}}^{\dagger}\right| 0\right\rangle \\
& =l_{B}(\mathbf{q} \times \overrightarrow{\mathscr{E}})_{z} .
\end{aligned}
$$

In principle, the second-order correction should be determined by the $K$-terms of the operator (17). By so calculating, attention should be given to the fact that the energy of the $\mathcal{Q}_{q}^{\dagger}|0\rangle$ state is counted off the ground state energy. However, the latter, for its part, also includes the second-order electrostatic correction, and both corrections appear to be equal. Thus, the difference vanishes and the $\propto \mathscr{E}^{2}$ total correction is reduced to a zero result. This feature is due to the symmetry of the purely electronic system considered. Formally, as applied to the case of a common two-particle magnetoexciton [9], this symmetry would correspond to electron and hole masses satisfying the $m_{e}=-m_{h}$ equivalence and thus result in vanishing of the $\sim \mathscr{E}^{2}$ term in the magnetoexciton energy. 
Now we can write out the relevant energy of the magnetoexciton within the domain. We consider its $q$ dispersion (2) in the vicinity of the weakly manifested roton minimum (see Ref. [帄). So, within a constant independent of parameters $\mathbf{q}$ and $\mathbf{R}$, the relevant part of the energy is

$$
\mathcal{E}^{\prime}(\mathbf{q}, \mathbf{R})=\alpha\left(q-q_{01}\right)^{2}+l_{B}(\mathbf{q} \times \overrightarrow{\mathscr{E}})_{z},
$$

where parameters $\alpha$ and $q_{01}$ are positive and supposedly estimated as $\alpha \simeq 0.1 \mathrm{meV}$ and $q_{01} \simeq 1$ in the $1 / l_{B}$ units. This energy reaches its local minimum value at

$$
\mathbf{q}=\mathbf{q}_{m}(\mathbf{R})=-\left(l_{B} / 2 \alpha+q_{01} / \mathscr{\mathscr { E }}\right) \overrightarrow{\mathscr{E}} \times \hat{z},
$$

which is the root of equation $\partial \varepsilon / \partial \mathbf{q}=0$. The local minimum corresponds to zero group velocity, hence to the zero drift velocity of the electron and effective 'hole' composing the magnetoexciton. Physically this means vanishing of the total electric field that acts on each quasiparticle since the electron-'hole' interaction field just compensates the external one. It is natural to consider the initial metastable state corresponding to this minimum. Due to cool down processes, diffusion and drift, that are fast compared to the CSFE lifetime, the magnetoexciton gets 'stuck' in the smooth random potential with energy $\delta^{(1)}+\varepsilon_{m}(\mathbf{R})$, where

$$
\mathcal{E}_{m}(\mathbf{R})=\mathcal{E}^{\prime}\left(\mathbf{q}_{m}, \mathbf{R}\right)=-l_{B} q_{01} \mathscr{E}-\left(\mathscr{E}_{B}\right)^{2} / 4 \alpha
$$

( $\delta^{(1)}$ is a constant independent of $\mathbf{R}$, see the study of spin-exciton kinetics in Ref. [12]). Thus, the system represents a frozen but chaotic state held by the smooth random potential. Magnetoexciton trapping occurs only in domains where $\left|\mathcal{E}_{m}\right| \gtrsim T$, hence, for $T \sim 1 \mathrm{~K}$ we get $l_{B} \mathcal{E} \gtrsim 1 \mathrm{~K}$ (i.e. $\mathscr{E} \gtrsim 100 \mathrm{~V} / \mathrm{cm})$. In principle, this is in agreement with the mean $\mathscr{E}$ value expected for the widethickness quantum well used in the experiment.

\subsection{Energy of the 'common' electron-hole magnetoexciton}

The final state $\left|v-e, \mathbf{q}_{m}\right\rangle$ emerging within the domain as a result of photon absorption is explicitly defined. It represents a 'common' two-particle 2D magnetoexciton studied, for instance, in Ref. [13] which is now, however, considered against the background of the zero Landau level completely occupied by conduction band electrons. This background is 'rigid' and its state, if calculated to the first order in the Coulomb interaction and external electric field, is not changed even in the presence of the magnetoexciton. The occupied electron Landau level for its part does not influence the $q$-dispersion of the energy $E_{v-e, \mathbf{q}}$ studied within the same approximation. In our specific case, where the valence-hole belongs to the zero Landau level and the electron to the first one, the $E_{v-e, q}$ energy represents a mode with the azimuthal number $m=1$ (the $E_{01}$ eigenstate in the notation of Ref. [13]). This mode has also a roton minimum, i.e. the actual $q$-dependence of the $E_{v-e, \mathbf{q}}$ energy in the vicinity of the $q_{m}$ value is $\sim \beta\left(q-q_{02}\right)^{2}$, where $q_{02} \sim 1$ and $\beta>0$. Now, if $q$-dispersion is considered with the use of the parabolic valence-band model, as in Refs. [9, 13], it is necessary to take into account the appropriate $v$-e effective form factor in the dispersion equation similar to Eq. (2). For values $q \gtrsim 1$ the $\mathcal{F}_{v e}(q)$ form factor is found to be considerably larger than the $\mathcal{F}_{e e}(q)$ one, i.e. it occurs owing to the greater 'compactness' of the heavy-hole size-quantized function $\left|\chi_{v}(z)\right|^{2}$ compared to that of the electron function $\left|\chi_{e}(z)\right|^{2}$. As a result, the property $\beta>\alpha$ holds for the $q$-dispersion parameters.

The energy dependence on external random field can be studied in the way described above with the only replacement of the field operator $\hat{\varphi}$ by the operator

$$
\hat{\varphi}-\overrightarrow{\mathscr{E}}(\mathbf{R}) \mathbf{r}_{h}
$$

where $\mathbf{r}_{h}$ is the position of the valence hole within the domain. The first-order correction in the electric field $\mathscr{E}$ is the same as for the purely electronic magnetoexciton, while the second order correction does not vanish. Indeed, first, the $\propto \mathscr{E}^{2}$ corrections to the energies of purely electronic states $b^{\dagger}|0\rangle$ and $|0\rangle$ determined by the operators of Eq. (19) do not compensate each other, unlike the case with states $\mathcal{Q}_{\mathbf{q}}^{\dagger}|0\rangle$ and $|0\rangle$. Second, the $\propto \mathscr{E}^{2}$ correction to the state $V_{p}^{\dagger}|0\rangle$ should also be taken into account. As a result, the total correction is the same as that found by Gor'kov and Dzyaloshinsky [9]. Finally, the relevant part of the $E_{v-e, \mathbf{q} m}$ energy is 


$$
\begin{aligned}
& \mathcal{E}_{v-e}\left(\mathbf{q}_{m}, \mathbf{R}\right)=\beta\left(q_{m}-q_{02}\right)^{2}+l_{B}\left(\mathbf{q}_{m} \times \overrightarrow{\mathscr{E}}\right)_{z} \\
& -\frac{\mathscr{E}^{2} l_{B}^{2}}{2 \hbar}\left(1 / \omega_{\mathrm{c}}^{(e)}+1 / \omega_{\mathrm{c}}^{(h)}\right),
\end{aligned}
$$

where $\beta>\alpha, q_{02} \sim 1 ; \omega_{c}^{(e)}$ and $\omega_{c}^{(h)}$ are the cyclotron frequencies in the conduction and valence bands, respectively. The last term in Eq. (22) is definitely small and, within the framework of the absorption mechanism studied here, it has to be taken into account only in the symmetric case where $\alpha \approx \beta$ and $q_{01} \approx q_{02}$. However, this term also becomes essential in further calculations relating to a coherent ensemble of magnetoexcitons.

\subsection{Estimate of the absorption rate according to formula (15)}

The domains participating in summation (15) must satisfy two conditions: (i) they contain a magnetoexciton and (ii) correspond to a vanishing argument of the $\delta$ function. Therefore, within the constant $C$ independent of the coordinate, we come to the equation

$$
\hbar \omega-C=\mathcal{E}_{v-e}\left(\mathbf{q}_{m}, \mathbf{R}\right)-\mathcal{E}_{m}(\mathbf{R})
$$

for R. Obviously, the first-order electrostatic terms $l_{B}\left(\mathbf{q}_{m} \times \overrightarrow{\mathscr{E}}\right)_{z}$ cancel each other in this difference. As a result, by substituting expressions (21) and (22), Eq. (23) can be rewritten in the form

$$
F(\mathbf{R})=\hbar \omega-\text { const, }
$$

where

$$
F(\mathbf{R})=\frac{\beta l_{B}}{\alpha}\left(q_{01}-q_{02}\right) \mathscr{E}+(\beta-\alpha) \frac{\left(\mathscr{E} l_{B}\right)^{2}}{4 \alpha^{2}},
$$

and const is a combination of the forbidden gap and the Coulomb, Zeeman and cyclotron shifts relevant to the case $\left(\propto \mathscr{E} / \omega_{c}^{(e, h)}\right.$ terms are ignored). The frequency $\omega$ of the probing laser beam seems to be appropriately tuned in order to ensure a maximum signal, i.e. specifically, in terms of our study, to provide the maximum number of domains participating in the sum (15). The solution of Eq. (24) determines a certain line in the $(x$, $y)$ plane. We introduce local orthogonal coordinates $s$ and $u$, where $s$ is the length along the line, and the unit vector $\hat{u}$ is parallel to $\nabla F$. It is apparent that in the general case of the smooth and random field $F(\mathbf{R})$, which is related to smoothness and randomness of $\mathscr{E}$, the line represents a closed non-self-intersecting curve (loop) with a length determined by the inhomogeneity characteristic $\Lambda$ and frequency $\omega$. First, consider a certain domain $\mathrm{d} u \times \mathrm{d} s$ adjacent to the curve. If the distribution of magnetoexcitons over the area $S=2 \pi l_{B}^{2} \mathcal{N}_{\phi}$ of the light spot created by the pumping laser is equiprobable, then the probability to find a CSFE within the domain is $(N / S) \mathrm{d} u \mathrm{~d} s$ and the contribution of the domain to the sum in Eq. (15) is $(N / S) \mathrm{d} s \int \delta(|\nabla F| \mathrm{u}) \mathrm{d} u=N \mathrm{~d} s / S|\nabla F|$. Summating over all such domains we estimate the contribution of a single loop to the sum $(15)$ as $N \mathcal{L}_{\omega} / S \mid \overline{\nabla F \mid}$, where $\mathcal{L}_{\omega}$ is the loop length and $|\overline{\nabla F}|$ is the mean value along the loop. It is natural to assume that at frequency $\omega$ corresponding to the maximum of the absorption signal the largest contribution to the (15) signal is provided by 'standard' loops (see the discussion on electron-drift trajectories in a quantum Hall system presented in Ref. [14]). For those we estimate $\mathcal{L}_{\omega} \sim \pi \Lambda^{\prime}$, where $\Lambda^{\prime}$ is the linear characteristic of the inhomogeneity for the $\mathscr{E}=|\nabla \varphi|$ field, and, hence, $\Lambda^{\prime} \sim \Lambda$. A more delicate estimate shows that $\Lambda^{\prime}=k \Lambda$, where $k<1$. Indeed, for example, if it is assumed that $\mathscr{E} \simeq \sqrt{\overline{(\nabla \varphi)^{2}}}$ and $\varphi$ is a Gaussian random field, then $\overline{|\nabla \varphi|^{2}}=2(\Delta / \Lambda)^{2}$, $\Delta$ describing potential amplitude $\left(\left(\overline{\varphi^{2}}\right)^{1 / 2}\right.$. Therefore, in this case $\mathscr{E} \simeq \sqrt{2} \Delta / \Lambda$, and $k \simeq 1 / \sqrt{2}$.

By analogy we find $\overline{|\nabla \mathscr{E}|} \simeq \Delta / \Lambda^{\prime 2}$ and $\overline{|\nabla \mathscr{E}|} \simeq \Delta^{2} / \Lambda^{\prime 3}$.

These estimates should be substituted into $|\nabla F| \sim(\beta / \alpha) l_{B}\left|q_{01}-q_{02}\right||\overline{\nabla \varepsilon \mid}+(\beta-\alpha)| \overline{\nabla \varepsilon^{2}} \mid l_{B}^{2} / 4 \alpha^{2}$. The last step in performing the summation in Eq. (15) is calculation of the total number of standard loops corresponding to the frequency $\omega$. Intuitively, this number is $\gamma S /\left(\pi \Lambda^{\prime 2} / 4\right)$ with the factor $\gamma \simeq 1 / 4$ for standard loops. Multiplying it by the contribution of one standard loop found above, we obtain an estimate for the absorption rate $\mathcal{R}_{\mathrm{I}}=\mathcal{K}_{\mathrm{I}} N$, where $\mathcal{K}_{\mathrm{I}}$ is the 'oscillator strength':

$$
\mathcal{K}_{\mathrm{I}}=2 \pi|A|^{2} / \hbar \Phi \Delta .
$$

In this formula

$$
\Phi \sim\left|q_{01}-q_{02}\right| \frac{\sqrt{2} \beta l_{B}}{\alpha \Lambda}+\left(\frac{\beta}{\alpha}-1\right) \frac{\Delta l_{B}^{2}}{2 \alpha \Lambda^{2}} .
$$


Since $\alpha, \beta$ and $l_{B}$ are inversely proportional to the square root of the magnetic field $B$, the oscillator strength (26) should grow with $B$.

\section{Light absorption by coherent magnetoexcitons}

The employed single-exciton approximation fails with growing magnetoexciton density $n=N / S$. We discuss the dependence of oscillator strength on density $n$ at the temperature which is certainly assumed to be lower than the value that enables to consider the incoherent magnetoexciton system as a frozen spatial chaos. Two scenarios of the influence of the inter-exciton interaction on oscillator strength may be assumed. The first represents a gradual evolution: the larger the magnetoexciton density is, the smoother the effective random potential becomes since the increasing density apparently results in a larger effective correlation length $\Lambda$. Indeed, dipole momenta $d_{\mathbf{q}_{m}}$ (9) oriented to minimise electrostatic energy should also create in the $2 \mathrm{D}$ space a screening electric field reducing the external one. The absorption signal should grow with weakening of the random electric field $\mathscr{E} \sim \Delta / \Lambda$ (see Eqs. (26) and (27)).

In the other scenario that we consider in more detail the oscillator strength increases abruptly at a certain value of $n=n_{c}$. This increase can be explained by a spontaneous rearrangement of the magnetoexciton system. We do not study the origin and features of this 'phase transition' definitely related to the inter-magnetoexciton interaction and favourable for occurrence of a coherent state. So, throughout this section as well as the previous one, we will stay within the framework of a model ignoring the inter-magnetoexciton interaction.

We demonstrate how the light absorption rate can be estimated within the framework of the model of a coherent state where a great number of magnetoexcitons belong to the same state, i.e. they have equal wave vectors. First, we consider a cluster with the area $\mathcal{L} \times \mathcal{L}$ (so that $\mathcal{N}_{\phi}=\mathcal{L}^{2} / 2 \pi l_{B}^{2}$ ) where all $N$ excitons in the cluster form a single coherent state. Now, instead of the initial state (10), we have

$$
|\tilde{N}\rangle=\left(\mathcal{Q}_{\mathbf{q}}^{\dagger}\right)^{N}|0\rangle
$$

The energy $E_{N q}$ of this state does not depend on any spatial fluctuations of the electrostatic field in the case of a large cluster size: $\mathcal{L} \gg \Lambda$. Indeed, at constant $\mathbf{q}$ the summation of electrostatic contributions $l_{B}(\mathbf{q} \times \hat{z}) \nabla \varphi(\mathbf{r})$ over the cluster area is reduced to the integration $\propto \int \mathrm{d} \mathbf{r} \nabla \varphi(\mathbf{r})$ and thereby yields a zero result. However, if $\mathcal{L} \lesssim \Lambda$, the electrostatic energy still contributes to $E_{N, \mathbf{q}}$. The squared norm of state (28) is calculated in the same way as it was done earlier in the case of $\mathbf{q} \equiv 0$. The norm $R_{N}$ was first calculated for the $\mathbf{q} \equiv 0$ case in the work by Dzyubenko and Lozovik [15]. The calculation was based on certain commutation rules for $\mathcal{Q}_{0}^{\dagger}$-exciton operators. It can also be performed in a similar way based on the commutation rules for the $\mathcal{Q}_{\mathrm{q}}^{\dagger}$ operators at arbitrary $\mathbf{q}$ (see the Appendix) and yielding the same formula. The result

$$
R_{N}=\langle\tilde{N} \mid \tilde{N}\rangle=N ! \mathcal{N}_{\phi} ! / \mathcal{N}_{\phi}^{N}\left(\mathcal{N}_{\phi}-N\right) !
$$

does not depend on q. Now we find the result of action of $\hat{\mathcal{P}}$ (see Eq. (12)) on the initial state: $\hat{\mathcal{P}}|\tilde{N}\rangle=-A N \hat{\chi_{\mathrm{q}}}|\widetilde{N-1}\rangle$. Within our approximation, ignoring any inter-excitonic coupling, we consider $\left|f_{\mathbf{q}}\right\rangle=\hat{\chi}_{\mathbf{q}}|\widehat{N-1}\rangle$ as a final state that has a squared norm equal to $R_{N-1}$ and energy $E_{N-1 q}+E_{v-e}$ (see Eq. (22) for $\mathcal{E}_{v-e}$ ), and thus calculate the transition matrix element squared

$$
\left|M_{N}\right|^{2}=\left|\left\langle f_{\mathbf{q}}|\hat{\mathcal{P}}| \tilde{N}\right\rangle\right|^{2} / R_{n} R_{N-1} \approx|A|^{2} N
$$

(the $N \ll \mathcal{N}_{\phi}$ condition is used). This result is by factor $N$ larger than $\left|M_{i}\right|^{2}$ found in the above calculation. Eq. (30) corresponds to the amplifcation by factor $N$ (and thereby also amplified radiation in resonance-reflection optics) for systems of coherent quantum oscillators (cf. spontaneous radiation by molecular gas [16]). However, the comparative absorbing capacity must again be estimated by calculating the oscillator strength, and again we are forced to take into account the external random field.

Indeed, according to Eq. (22), the energy of the $\chi_{\mathbf{q}}|0\rangle$ exciton depends on the field $\mathscr{E}(\mathbf{R})$. We divide the cluster area $\mathcal{L} \times \mathcal{L}$ into small domains parameterised by coordinate $\mathbf{R}$, and therefore again consider $\mathscr{E}(\mathbf{R})$ and $\nabla \mathscr{E}(\mathbf{R})$ within every domain as constant parameters. (The linear dimension of the domain is assumed to be smaller than $\Lambda$ but larger than $l_{B}$.) Then the matrix element for transition resulting in creation of a $\chi_{\mathbf{q}}|0\rangle$ exciton within the R-domain is determined by Eq. (30) with $N$ 
replaced by $\rho \mathrm{dR}$. ( $\rho=N / \mathcal{L}^{2}$ to describe the CSFE density in the cluster considered to be constant.) Thus, the absorption rate represents a sum over all domains, i.e. integration over the $2 \mathrm{D}$ space:

$$
\begin{aligned}
& \mathcal{R}_{\mathrm{II}}=\frac{2 \pi|A|^{2} \rho}{\hbar} \\
& \int \mathrm{d} \mathbf{R} \delta\left(\hbar \omega+E_{N, \mathbf{q}}-E_{N-1 \mathbf{q}}-E_{v-e, \mathbf{q}}\right) .
\end{aligned}
$$

Considering the difference $E_{N \mathbf{q}}-E_{N-1 \mathbf{q}}-\mathcal{E}_{v-e}(\mathbf{q}, \mathbf{R})$ within the domain (see Eq. (22) for $\mathcal{E}_{v-e}$ ) we conclude that the first-order electrostatic terms $l_{B}(\mathbf{q} \times \hat{z}) \mathscr{E}(\mathbf{R})$ compensate each other in the initial and final states, and therefore do not enter the difference. Now, however, the electrostatic contribution to the argument of the $\delta$ function in Eq. (31) is related to the $\propto \mathscr{E}^{2}$ terms in Eq. (22). The situation differs from the previous one in the replacement of field $F(\mathbf{R})$ with field $-\mathscr{E}^{2}(\mathbf{R})\left(1 / \omega^{(e)}+1 / \omega_{c}^{(h)}\right) l_{B}^{2} / 2 \hbar$. Considering again the $(u, v)$ local coordinate system $(\mathrm{d} \mathbf{R}=\mathrm{d} u \mathrm{~d} v)$ we choose the unit vector $\hat{u}$ directed along the gradient $\nabla \mathscr{E}^{\mathscr{2}}(\mathbf{R})$. As a result, estimating $\left|\nabla \mathscr{E}^{2}\right| \sim \Delta^{2} / \Lambda^{\prime 3}$ and following Eq. (31), we find, first, the contribution of one 'standard' loop to the absorption rate. And, finally, multiplying by the number of standard loops within the cluster $\sim \mathcal{L}^{2} / \pi \Lambda^{\prime 2}$ (assuming $\mathcal{L} \gg \Lambda$ ), we find the contribution of the cluster to the absorption rate $\mathcal{R}_{\mathrm{II}}=\mathcal{K}_{\mathrm{II}} N$, where the oscillator strength is

$$
\mathcal{K}_{\mathrm{II}} \sim 2|A|^{2} \pi \omega_{\mathrm{c}}^{(h)}\left(\Lambda / l_{B} \Delta\right)^{2}
$$

(considering that $\omega_{c}^{(h)}<\omega_{c}^{(e)}$ and $\Lambda^{\prime} \simeq \Lambda / \sqrt{2}$ ). Thus, the enhancement of the absorption/reflection signal due to magnetoexciton clustering is $\mathcal{K}_{\mathrm{II}} / \mathcal{K}_{\mathrm{r}}$. In the case where the first term in Eq. (27) is assumed to be larger than the second one we obtain

$$
\mathscr{K}_{\mathrm{II}} / \mathscr{K}_{\mathrm{I}} \sim \frac{\hbar \omega_{\mathrm{c}}^{(h)} \beta \Lambda\left|q_{01}-q_{02}\right|}{\alpha l_{B} \Delta} .
$$

Using the actual experimental data [6]: $\Delta / \hbar \omega_{c}^{(h)} \simeq 0.1, \alpha / \beta \simeq 0.5, l_{B} / \Lambda \simeq 0.2$ and $\left|q_{01}-q_{02}\right| \simeq 0.1$, we obtain the estimate

$$
\mathcal{K}_{\mathrm{II}} / \mathcal{K}_{\mathrm{I}} \simeq 10 .
$$

So, when studying light absorption/reflection, we expect the amplification effect to be approximately by an order of magnitude in the case of quantum transition between incoherent and coherent phases.

\section{Long-distant Coulomb correlations in a slightly non-ideal magnetoexcitonic gas}

Now, in contrast to the previous sections, we will neglect any disorder but take into account a weak inter-exciton coupling. So, considering our system in the 'clean limit', we will study deviation of an actual excitonic gas from the ideal state due to double exciton correlations. We will also ignore the probability of any essential change of $\mathcal{Q}_{\mathrm{q} i}^{\dagger}|0\rangle$ single exciton quantum states, including, for instance, formation of bi-excitons or dissociations into electron-hole pairs. It will enable us to find only a small correction to the single CSFE energy proportional to the small but finite concentration $N / \mathcal{N}_{\phi}$. This first-order virial correction can be compared to the correction related to the smooth random potential studied above.

\subsection{Double exciton state}

Within the first-order approximation in terms of a small parameter $r_{\mathrm{s}}(1)$ the single magnetoexciton state $\mathcal{Q}_{\mathrm{q}}^{\dagger}|0\rangle$ diagonalizes the Hamiltonian $\hat{H}_{\text {Coul }}$ (see Eq. (8)), but the double exciton state $\mathcal{Q}_{\mathrm{q}_{1}}^{\dagger} \mathcal{Q}_{\mathrm{q}_{2}}^{\dagger}|0\rangle$ does not. The latter is an 'almost' eigenstate. Indeed, we have

$$
\begin{aligned}
& {\left[\hat{H}_{\mathrm{Coul}}, \mathcal{Q}_{\mathbf{q}_{1}}^{\dagger} \mathcal{Q}_{\mathbf{q}_{2}}^{\dagger}\right]|0\rangle=\left(\mathcal{E}_{q_{1}}+\mathcal{E}_{q_{2}}\right) \mathcal{Q}_{\mathbf{q}_{1}}^{\dagger} \mathcal{Q}_{\mathbf{q}_{2}}^{\dagger}|0\rangle} \\
& +\left[\left[\hat{H}_{\mathrm{Coul}}, \mathcal{Q}_{\mathbf{q}_{1}}^{\dagger}\right], \mathcal{Q}_{\mathbf{q}_{2}}^{\dagger}\right]|0\rangle,
\end{aligned}
$$

where the double-commutation term arises as a result of the magnetoexciton interaction. We emphasise that by calculating the commutators in Eq. (34), as well as in Eq. (8), as part of our approximation approach, in the $\hat{H}_{\text {Coul }}$ operator it is possible to keep only the terms preserving the cyclotron energy of the ket vector $\mathcal{Q}_{\mathbf{q}_{1}}^{\dagger} \mathcal{Q}_{\mathbf{q}_{2}}^{\dagger}|0\rangle$. This simplifies the calculation by changing from $\hat{H}_{\text {Coul }}$ to $\hat{H}_{\text {Coul }}^{\prime}$ (see Eq. (A7) for $\hat{H}_{\text {Coul }}$ in the Appendix), so that the exciton-exciton interaction results in

$$
\begin{aligned}
& {\left[\left[\hat{H}_{\text {Coul }}^{\prime}, \mathcal{Q}_{\mathbf{q}_{1}}^{\dagger}\right], \mathcal{Q}_{\mathbf{q}_{2}}^{\dagger}\right]} \\
& =\sum_{\mathbf{q}} \frac{\mathcal{F}_{e e}(q)}{q \mathcal{N}_{\phi}}\left(h_{00 \mathbf{q}} \mathrm{e}^{\mathrm{i} \mathbf{q} \times \mathbf{q}_{1} / 2}-h_{11 \mathbf{q}} \mathrm{e}^{\mathrm{i} \mathbf{q}_{1} \times \mathbf{q} / 2}\right) \\
& \times\left(h_{00 \mathbf{q}} \mathrm{e}^{\mathrm{i} \mathbf{q}_{2} \times \mathbf{q} / 2}-h_{11 \mathbf{q}} \mathrm{e}^{\mathrm{iq} \times \mathbf{q}_{2} / 2}\right) \mathcal{Q}_{\mathbf{q}_{1}+\mathbf{q}}^{\dagger} \mathcal{Q}_{\mathbf{q}_{2}-\mathbf{q}}^{\dagger},
\end{aligned}
$$


where functions $h \ldots$, are given by Eq. (A2) in the Appendix. It is interesting that, unlike the $\left[\hat{H}_{\text {Coul }}^{\prime}, \mathcal{Q}_{\mathbf{q}}^{\dagger}\right]$ commutator that does not commute with the exciton operator, the double-commutator (35) commutes with any operator $\mathcal{Q}_{\mathrm{q}}^{+}$. This means that triple, quadruple, etc. commutators are equal to zero. In order to find the first-order Coulomb correction to the energy, the state (35) should be projected onto the unperturbed doubleexciton state $\mathcal{Q}_{\mathbf{q}_{1}}^{\dagger} \mathcal{Q}_{\mathbf{q}_{2}}^{\dagger}|0\rangle$. Considering that

$$
\begin{aligned}
& \left\langle 0\left|Q_{\mathbf{q}_{2}} Q_{\mathbf{q}_{1}}\right| Q_{\mathbf{q}_{1}+\mathbf{q}}^{\dagger} Q_{\mathbf{q}_{2}-\mathbf{q}}^{\dagger} \mid 0\right\rangle=\delta_{\mathbf{q}, 0} \\
& +\delta_{\mathbf{q}_{\mathbf{q}} \mathbf{q}_{2}-\mathbf{q}_{1}}-\frac{\mathrm{e}^{\mathrm{i} \mathbf{q} \times\left(\mathbf{q}_{1}-\mathbf{q}_{2}\right) / 2}+\mathrm{e}^{\mathrm{i}\left(\mathbf{q}_{1}-\mathbf{q}_{2}\right) \times \mathbf{q} / 2}}{\mathcal{N}_{\phi}}
\end{aligned}
$$

(see Eq. (A8) in the Appendix) we obtain a correction to the double exciton energy $\mathcal{E}_{q 1}+\mathcal{E}_{q 2}$ proportional to $1 / \mathcal{N}_{\phi}$, namely:

$$
\begin{aligned}
& \left\langle 0\left|\mathcal{Q}_{\mathbf{q}_{2}} \mathcal{Q}_{\mathbf{q}_{1}}\left[\left[\hat{H}_{\text {Coul }}^{\prime}, \mathcal{Q}_{\mathbf{q}_{1}}^{\dagger}\right], \mathcal{Q}_{\mathbf{q}_{2}}^{\dagger}\right]\right| 0\right\rangle \\
& =\left[W_{1}\left(\mathbf{q}_{1}, \mathbf{q}_{2}\right)+W_{2}\left(\mathbf{q}_{1}, \mathbf{q}_{2}\right)\right] / \mathcal{N}_{\phi} .
\end{aligned}
$$

Here the function $W_{1}(\ldots)$ comes from the second Kronecker delta in Eq. (36) (the first one, $\delta_{\mathbf{q}, 0}$, yields a zero contribution to the result). Designating $q_{12}^{2}=\left|\mathbf{q}_{1}-\mathbf{q}_{2}\right|^{2}=q_{1}^{2}+q_{2}^{2}-2 q_{1} q_{2} \cos \Phi$, we get

$$
\begin{aligned}
& W_{1}\left(\mathbf{q}_{1}, \mathbf{q}_{2}\right)=\frac{\mathcal{F}_{e e}\left(q_{12}\right)}{q_{12}} \mathrm{e}^{-q_{12}^{2} / 2}\left[\frac{q_{12}^{4}}{4}\right. \\
& \left.+\left(4-2 q_{12}^{2}\right) \sin ^{2}\left(\frac{q_{1} q_{2} \sin \Phi}{2}\right)\right] .
\end{aligned}
$$

The function $W_{2}(\ldots)$ arises from the last term in Eq. (36) and contains the summation over $\mathbf{q}$ in accordance with Eq. (35). The summation is certainly converted to the integration that can be performed over the directions of vector $\mathbf{q}$, then we arrive at

$$
\begin{aligned}
& W_{2}\left(\mathbf{q}_{1}, \mathbf{q}_{2}\right) \\
& =\int_{0}^{\infty} \mathrm{e}^{-q^{2} / 2} \mathrm{~d} q \mathcal{F}_{e e}(q)\left\{\left(2-q^{2}\right)\left[J_{0}\left(q q_{1}\right)+J_{0}\left(q q_{2}\right)\right]\right. \\
& \left.-\left(2-q^{2}+q^{4} / 4\right)\left[1+J_{0}\left(q q_{12}\right)\right]\right\}
\end{aligned}
$$

This correction to the double-exciton energy proportional to $1 / \mathcal{N}_{\phi}$ appears because every mag- netoexciton is localised within the finite area $2 \pi l_{B}^{2} \mathcal{N}_{\phi}$. At first glance, the found correction is found with a 'superfluous accuracy', since single exciton energy is also determined only with accuracy to the $\sim E_{\mathrm{C}} / \mathcal{N}_{\phi}$ value. However, our result (37)-(39) appears only due to the exciton-exciton correlation and, hence, for $N \gg 1$ magnetoexcitons this correction becomes proportional to $N^{2} / \mathcal{N}_{\phi}$ (see the next subsection), whereas the error in theenergyof $N$ non-interactingexcitonsis $\sim N E_{C} / \mathcal{N}_{\phi}$. Another feature of the found correction is its finiteness even if both momenta $\mathbf{q}_{1}$ and $\mathbf{q}_{2}$ become zero. This property of the $W_{2}$ value is related to the fact that the electron and effective hole forming the studied magnetoexciton belong to different Landau levels. Therefore, the magnetoexciton essentially changes the orbital wave function of the 2DEG compared to the ground state even in the $\mathbf{q}=0$ case: in other words, the $\mathbf{q}=0$ CSFE having no dipole moment still has a quadrupole moment. This feature distinguishes the CSFE from, for instance, the spin-wave exciton in the $v<2$ quantum Hall system, where the electron and effective hole belong to the same Landau level, and in the $\mathbf{q}=0$ case this excitation does not perturb the 2DEG orbital state.

For equal absolute values, $\left|\mathbf{q}_{1}\right|=\left|\mathbf{q}_{2}\right|=q$, the dependence of the sum $U(q, \Phi)=W_{1}+W_{2}$ is shown in Fig. 1(a). So, the long-distance magnetoexciton interaction is actually attractive. There is a deep minimum at $\Phi=0$, i.e. in case magnetoexciton momenta are aligned along the same direction. In Fig. 1 (b) we also demonstrate the dependence of the function $V(p)=W_{1}+W_{2}$, where the momenta are parallel but not equal, namely: $\Phi=0$, $\left|\mathbf{q}_{1}\right|=q_{01}+p$ and $\left|\mathbf{q}_{2}\right|=q_{01}-p$ with the fixed parameter $q_{01}$ corresponding to the minimum of the single-exciton energy dispersion $\mathcal{E}_{q}$.

Note that in the special coherent state with $\mathbf{q}_{1} \equiv \mathbf{q}_{2}$ the result of calculation (37) must be divided by the squared norm of the state $\left(\mathcal{Q}_{\mathrm{q}}^{\dagger}\right)^{2}|0\rangle$, which is not unit now but equal to $R_{2}=2$. This doubleexciton 'coherent' state is a dedicated point marked by closed dots in Fig. 1 (a, b).

\subsection{Multi-excitonic state. Virial correction to magnetoexciton energy}

Now we study again the $|N\rangle$ multi-excitonic state (see Eq. (10)) with the exciton number $N$ that is 


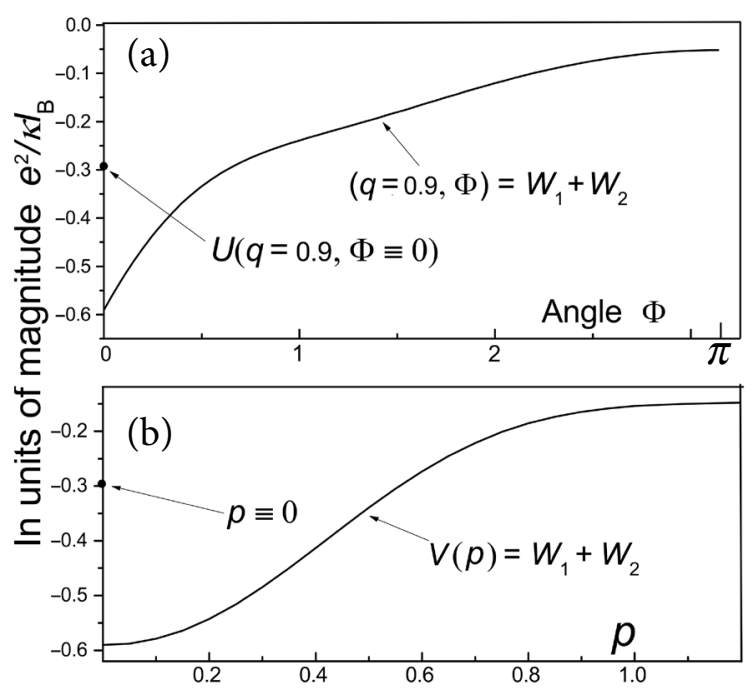

Fig. 1. Functions $U(q, \Phi)$ and $V(p)$ measured in units of $e^{2} / \kappa l_{B}$ (see the text). Both are calculated at the parameter $d=1$ with the form factor given by Eq. (4). Dependence of $U(q, \Phi)$ on the angle $\Phi$ between $\mathbf{q}_{1}$ and $\mathbf{q}_{2}$ is shown for the specific value $q=\left|\mathbf{q}_{1}\right|=\left|\mathbf{q}_{2}\right|=0.9$ (a). Function $V(p)=W_{1}+W_{2}$, where $\Phi=0$, is calculated for $q_{1}=0.9+p$ and $q_{2}=0.9-p$ (b). The 'coherent' case $\mathbf{q}_{1} \equiv \mathbf{q}_{2}$ is marked by closed dots.

much smaller than $\mathcal{N}_{\phi}$. This condition allows us to consider not only all $\mathbf{q}$ momenta to be different but the momentum of any pair $\mathbf{q}_{i}+\mathbf{q}_{j}$ different from any other pair momentum $\mathbf{q}_{i}^{\prime}+\mathbf{q}_{j}^{\prime}$. We introduce the notation

$$
\mathscr{Q}_{i, \kappa}^{\dagger}=\mathcal{Q}_{\mathbf{q}_{i}}^{\dagger} \mathcal{Q}_{\mathbf{q}_{i+1}}^{\dagger} \ldots \mathcal{Q}_{\mathbf{q}_{\kappa-1}}^{\dagger} \mathcal{Q}_{\mathbf{q}_{\kappa}}^{\dagger},
$$

where $\kappa \geq i$ and, besides, define that $\mathscr{Q}_{i, i-1}^{\dagger}=1$. In particular, $|N\rangle=\mathscr{Q}_{i, N}^{\dagger}|0\rangle$. We present the action of operator $\hat{H}_{\text {Coul }}$ as

$$
\begin{aligned}
& \hat{H}_{\mathrm{Coul}}|N\rangle=E_{\mathrm{Coul}}^{(0)}|N\rangle \\
& +\sum_{i=1}^{N} \mathscr{Q}_{1, i-1}^{\dagger}\left[\hat{H}_{\mathrm{Coul}}, \mathcal{Q}_{\mathbf{q} i}^{\dagger}\right] \mathscr{Q}_{i+1, N}^{\dagger}|0\rangle,
\end{aligned}
$$

where $E_{\text {Coul }}^{(0)}$ is the Coulomb part of the ground state energy. The summation in Eq. (40) is converted to

$$
\begin{aligned}
& \sum_{i=1}^{N} \mathscr{Q}_{1, i-1}^{\dagger} \mathscr{Q}_{i+1, N}^{\dagger}\left[\hat{H}_{\mathrm{Coul}}, \mathcal{Q}_{\mathbf{q}_{i}}^{\dagger}\right]|0\rangle \\
& +\sum_{i<j} \mathscr{Q}_{1, i-1}^{\dagger} \mathscr{Q}_{i+1, j-1}^{\dagger}\left[\left[\hat{H}_{\mathrm{Coul}}, \mathcal{Q}_{\mathbf{q}_{i}}^{\dagger}\right], \mathcal{Q}_{\mathbf{q}_{j}}^{\dagger}\right] \mathscr{Q}_{j+1, N}^{\dagger}|0\rangle .
\end{aligned}
$$

In the first approximation of the interaction we can again change from $\hat{H}_{\text {Coul }}$ to $\hat{H}_{\text {Coul }}^{\prime}$, then find the first sum in Eq. (41),

$$
\begin{aligned}
& \sum_{i=1}^{N} \mathscr{Q}_{1, i-1}^{\dagger} \mathscr{\mathscr { O }}_{i+1, N}^{\dagger}\left[\hat{H}_{\mathrm{Coul}}^{\prime}, \mathcal{Q}_{\mathbf{q}_{i}}^{\dagger}\right]|0\rangle \\
& =\left(\mathcal{E}_{q_{1}}+\mathcal{E}_{q_{2}}+\ldots+\mathcal{E}_{q_{N}}\right)|N\rangle,
\end{aligned}
$$

and present the second sum containing double commutators as

$$
\sum_{i<j} \mathscr{T}_{1, i-1}^{\dagger} \mathscr{Q}_{i+1, j-1}^{\dagger} \mathscr{Q}_{j+1, N}^{\dagger}\left[\left[\hat{H}_{\mathrm{Coul}}^{\prime}, \mathcal{Q}_{\mathbf{q}_{i}}^{\dagger}\right], \mathcal{Q}_{\mathbf{q}_{j}}^{\dagger}\right]|0\rangle .
$$

Sums with triple commutators and with commutators of higher orders are absent as a result of vanishing (see above). Sum (43) with double commutators contains $\approx N^{2} / 2$ terms. In order to find the appropriate contribution to the total energy this sum should be projected to the $|N\rangle$ state. After a simple analysis based on the commutation algebra of the exciton operators we find this projection in the leading approximation in terms of a small parameter $\bar{n}=N / \mathcal{N}_{\phi}$ :

$$
\approx \sum_{i<j}\langle N-2 \mid N-2\rangle\left\langle 0\left|\mathcal{Q}_{\mathbf{q}_{j}} \mathcal{Q}_{\mathbf{q}_{i}}\left[\left[\hat{H}_{\text {Coul }}^{\prime}, \mathcal{Q}_{\mathbf{q}_{i}}^{\dagger}\right], \mathcal{Q}_{\mathbf{q}_{j}}^{\dagger}\right]\right| 0\right\rangle .
$$

Then dividing by the norm $\langle N \mid N\rangle$ and using Eqs. (11) and (37) we obtain the sum of all the terms giving contribution of the inter-exciton interaction to the total Coulomb energy:

$$
\frac{1}{\mathcal{N}_{\phi}} \sum_{i<j}\left[W_{1}\left(\mathbf{q}_{i}, \mathbf{q}_{j}\right)+W_{2}\left(\mathbf{q}_{i}, \mathbf{q}_{j}\right)\right] .
$$

This sum is of the order of $N^{2} \mathcal{E}_{q} / \mathcal{N}_{\phi}$. To find its specific value it is necessary to know the distribution of the $\mathbf{q}_{i}$ vectors in the $K$-space determined by temperature and smooth random potential. Let us consider the simple case where temperature is equal to zero, and, hence, all $q_{i}$ have approximately the same absolute values, for instance, in the vicinity of the dispersion minimum $q_{01}$, but simultaneously have an isotropic distribution of their directions. Then summation in Eq. (43) is reduced to averaging over the angle $\Phi$ between $\mathbf{q}_{i}$ and $\mathbf{q}_{j}, \sum_{i<j}^{N} \ldots=$ $N(N-1) \int_{\ldots} . . \mathrm{d} \Phi / 4 \pi$. Dividing this result by $N$, we obtain the correction to the energy of one magnetoexciton proportional to the magnetoexcitonic concentration $\bar{n}=N / \mathcal{N}_{\phi}$. So, if $\left|\mathbf{q}_{1}\right|=\left|\mathbf{q}_{2}\right|=\ldots=\left|\mathbf{q}_{N}\right|=q$, the correction is 


$$
\mathcal{E}_{\text {vir }}(q)=\bar{n}\left[w_{1}(q)+w_{2}(q)\right] .
$$

Here

$$
w_{1}(q)=\frac{1}{4 \pi} \int_{0}^{2 \pi} \mathrm{d} \Phi W_{1}\left(\mathbf{q}_{1}, \mathbf{q}_{2}\right),
$$

where $W_{1}$ is determined by formula (38) with $q_{1}=q_{2}=q$ and $q_{12}=2 q\left|\sin \frac{\Phi}{2}\right|$, and

$$
\begin{aligned}
& w_{2}(q)=\left.\frac{1}{4 \pi} \int_{0}^{2 \pi} \mathrm{d} \Phi W_{2}\left(\mathbf{q}_{1}, \mathbf{q}_{2}\right)\right|_{q_{1}=q_{2}=q} \\
& =\int_{0}^{\infty} \mathrm{e}^{-p^{2} / 2} \mathrm{~d} p \mathcal{F}_{e e}(p)\left\{\left(2-p^{2}\right) J_{0}(q, p)\right. \\
& \left.-\left(1-p^{2} / 2+p^{4} / 8\right)\left(1+\left[J_{0}(q p)\right]^{2}\right)\right\} .
\end{aligned}
$$

The function $\mathcal{E}_{\mathrm{vir}}(q) / \bar{n}=w_{1}(q)+w_{2}(q)$ calculated using the form factor (4) with $d=1$ is shown in Fig. 2(a). Thus, for actual values $0<q \lesssim 1$ the virial correction is negative.

Finally, we study the correction for the coherent state where all momenta are the same, i.e. $\mathbf{q}_{i} \equiv \mathbf{q}$. Again the condition $1 \ll N \ll \mathcal{N}_{\phi}$ is considered to be satisfied. Generally, the calculation scheme remains the same: the action of $\hat{H}_{\text {Coul }}$ onto $|\tilde{N}\rangle=\left(\mathcal{Q}_{\mathbf{q}}^{\dagger}\right)^{N}|0\rangle$ results in the double commutation term

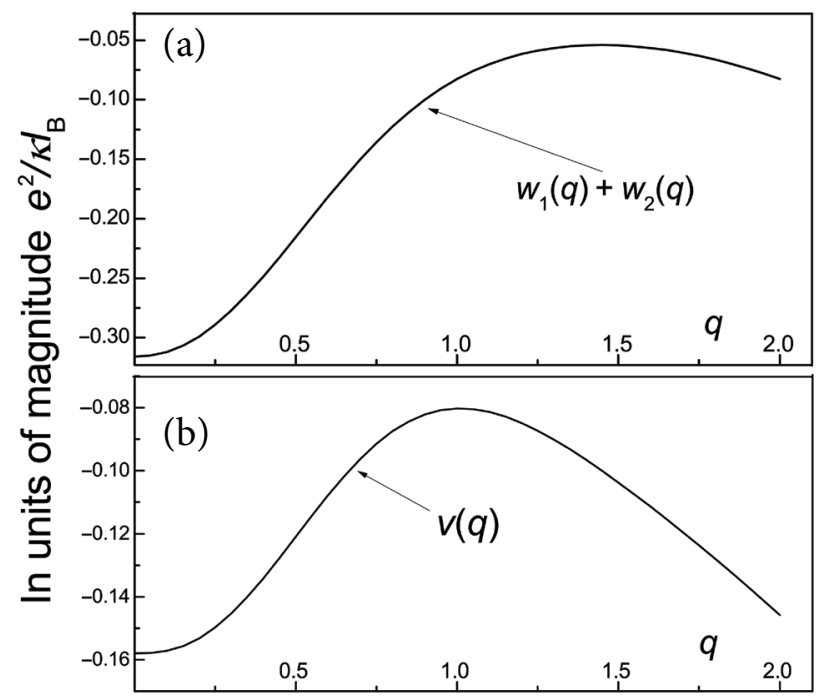

Fig. 2. Energies are measured in units of $e^{2}=\kappa l_{B}$ and calculated at parameter $d=1$ with the form factor given by Eq. (4). Virial correction in incoherent magnetoexcitonic gas (a): $\mathcal{E}_{\text {vir }}(q) / \bar{n}=w_{1}(q)+w_{2}(q)$ (see the text). Virial correction in the coherent state (b): $\mathcal{E}_{\text {vir }}(q) \bar{n}=v(q)$.

$$
\frac{N^{2}}{2}\left(\mathcal{Q}_{\mathbf{q}}^{\dagger}\right)^{N-2}\left[\left[\hat{H}_{\text {Coul }}^{\prime}, \mathcal{Q}_{\mathbf{q}}^{\dagger}\right], \mathcal{Q}_{\mathbf{q}}^{\dagger}\right]|0\rangle
$$

(cf. Eq. (43)). Then by using Eq. (35) and projecting onto $|\tilde{N}\rangle$ we obtain

$$
\begin{aligned}
& N^{2} \sum_{\mathbf{p}} \frac{\mathcal{F}_{e e}(p)}{2 p \mathcal{N}_{\phi}}\left[h_{00 \mathbf{p}}^{2}+h_{11 \mathbf{p}}^{2}\right. \\
& \left.-2 h_{00 \mathbf{p}} h_{11 \mathbf{p}} \cos (\mathbf{p} \times \mathbf{q})\right] P_{N \mathbf{p}},
\end{aligned}
$$

where

$$
P_{N \mathbf{p}}=\left\langle\tilde{N}\left|\left(\mathcal{Q}_{\mathbf{q}}^{\dagger}\right)^{N-2} \mathcal{Q}_{\mathbf{q}+\mathbf{p}}^{\dagger} \mathcal{Q}_{\mathbf{q}-\mathbf{p}}^{\dagger}\right| 0\right\rangle .
$$

In order to find the correction to energy this expression has to be divided by the squared norm $R_{N}$ (29). The analysis based on the commutation rules for $\mathcal{Q}$ operators shows that under our conditions

$$
P_{N \mathbf{p}} / R_{N}=\delta_{\mathbf{p}, 0}-\mathcal{N}_{\phi}^{-1}
$$

(see the Appendix). So, taking into account that $h_{000}=h_{110} \equiv 1$ and dividing expression (48) by $R_{N}$ and $N$ we obtain a virial correction per one magnetoexciton in the condensate state, $\widetilde{\mathcal{E}}_{\text {vir }}(q)=\bar{n} v(q)$, where

$$
\begin{aligned}
& v(q)=\int_{0}^{\infty} \mathrm{e}^{-p^{2} / 2} \mathrm{~d} p \mathcal{F}_{e e}(p)\left[\left(1-p^{2} / 2\right) J_{0}(p q)\right. \\
& \left.-1+p^{2} / 2-p^{4} / 8\right] .
\end{aligned}
$$

This is also negative, see Fig. 2(b).

\section{Conclusions}

In conclusion, we estimate the CSFE concentration $\bar{n}$ responsible for failing single-exciton corrections related to the external disorder. It is obvious that the interaction energy per one magnetoexciton calculated above should be compared to energy (21) holding the magnetoexciton by the smooth random potential. In the incoherent state, if $q \simeq 1$, virial correction (45) is $\mathcal{E}_{\text {vir }} \simeq-0.1 \bar{n}$ if measured in $e^{2} / \kappa l_{B}$ units. Considering magnetic fields in the range $B=5-10, l_{B} \simeq 10 \mathrm{~nm}$, and assuming $\mathcal{E} \simeq \Delta / \Lambda$ with $\Delta \simeq 0.5-0.8 \mathrm{meV}$ and $\Lambda=50 \mathrm{~nm}$, the comparison leads to an estimate for the critical concentration $\bar{n}_{\mathrm{c}} \simeq 0.1$ allowing excitation to escape from the local energy minimum. Besides, according to our study, this concentration should be basically inversely proportional to the field $B$. 
Thus, long-distant inter-excitonic correlations in a nonideal magnetoexciton gas are attractive, which should certainly contribute to some 'selforganization' of the system and to a transition from the incoherent to coherent phase. It is noteworthy that pair correlations are most significant for magnetoexcitons with co-directional momenta (see the $\Theta=0$ case in the results presented in Fig. 1). The effectively critical concentration for such excitations becomes considerably lower than the value found above. As a result, we should expect the usual pattern of transition through the inhomogeneous intermediate state, namely, via formation of coherent phase embryos, domain clusters, etc.

We emphasise once again that our calculations valid only for a weakly nonideal gas, in principle, do not exclude a different scenario of appearance of a dense phase in the system: for instance, an ensemble of bi-excitons or an electron-hole liquid may arise instead of a coherent state.

The research was supported by the Russian Science Foundation for Basic Research: Grant 1802-01064. The author is grateful to I.V. Kukushkin and L.V. Kulik for useful discussions.

\section{Appendix A}

\section{Excitonic representation (ER)}

The general expression for the Coulomb Hamiltonian

$$
\hat{H}_{\text {Coul }}=\frac{1}{2} \int \mathrm{d} \mathbf{r}_{1} \mathrm{~d} \mathbf{r}_{2} \hat{\Psi}^{\dagger}\left(\mathbf{r}_{1}\right) \hat{\Psi}^{\dagger}\left(\mathbf{r}_{2}\right) U\left(\mathbf{r}_{1}-\mathbf{r}_{2}\right) \hat{\Psi}\left(\mathbf{r}_{2}\right) \hat{\Psi}\left(\mathbf{r}_{1}\right),
$$

where $\hat{\Psi}(\mathbf{r})=\sum_{n p \sigma} c_{n p \sigma} \psi_{n p}(\mathbf{r})$ (see Eq. (6)), can be rewritten in the form

$$
\begin{aligned}
& \hat{H}_{\text {Coul }} \\
& =\frac{1}{2 \mathcal{N}_{\phi}} \sum_{\substack{p, p^{\prime}, \mathbf{q} \\
a, b, c, d}} V_{b d c a}(\mathbf{q}) \mathrm{e}^{\mathrm{i} q_{x}\left(p-p^{\prime}\right)} b_{p-q_{y}}^{\dagger} d_{p^{\prime}}^{\dagger} c_{p^{\prime}-q_{y}} a_{p} \cdot(\mathrm{A} 1)
\end{aligned}
$$

Here operators $a_{p}, b_{p}, c_{p}$ and $d_{p}$ are electron annihilation operators $c_{\text {npo }}$ corresponding to binary indexes $a, b, c$ and $d$ where each designates both the Landau level number and the spin sublevel. For example, $a=\left(n_{a}, \sigma_{a}\right)$. Factors

$$
V_{b d c a}(\mathbf{q})=\left[\mathcal{F}_{e e}(q) / q\right] h_{n_{a} n_{b}-\mathbf{q}{ }_{n_{c} n_{d} \mathbf{q}}} \delta_{\sigma_{a}, \sigma_{b}} \delta_{\sigma_{c} \sigma_{d}}
$$

are expressed in the formfactor $\mathcal{F}_{e e}$ and the building block functions used in the ER technique

$$
h_{k n \mathbf{q}}=\left(\frac{k !}{n !}\right)^{1 / 2} \mathrm{e}^{-q^{2} / 4}\left(q_{-}\right)^{n-k} L_{k}^{n-k}\left(q^{2} / 2\right) \text {, }
$$

where $q_{ \pm}= \pm \frac{\mathrm{i}}{\sqrt{2}}\left(q_{x} \pm \mathrm{i} q_{y}\right)$ and $L_{n}^{k}$ are the Laguerre polynomials. For its part, expression (A1) can be presented in terms of excitonic $\mathcal{Q}$-operators

$$
\begin{aligned}
& \hat{H}_{\text {Coul }}=\sum_{\mathbf{q}, a, b, c, d} \frac{\mathcal{F}_{e e}(q)}{2 q}\left(h_{n_{a} n_{b} \mathbf{q}} \delta_{\sigma_{a}, \sigma_{b}} Q_{a b \mathbf{q}}^{\dagger}\right) \\
& \times\left(h_{n_{c} n_{d}-\mathbf{q}} \delta_{\sigma_{c}, \sigma_{d}} Q_{c d-\mathbf{q}}^{\dagger}\right)-\sum_{\mathbf{q}, a, b} \frac{\mathcal{F}_{e e}(q)}{2 q}\left|h_{n_{a} n_{b}}(\mathbf{q})\right|^{2} \mathcal{B}_{0}^{\dagger},
\end{aligned}
$$

where

$$
Q_{a b \mathbf{q}}^{\dagger}=\mathcal{N}_{\phi}^{-1 / 2} \sum_{p} \mathrm{e}^{-\mathrm{i} q_{x} p} b_{p+q_{y} / 2}^{\dagger} a_{p-q_{y} / 2}
$$

(note that $\mathcal{Q}_{a b \mathrm{q}} \equiv \mathcal{Q}_{b a-\mathbf{q}}^{\dagger}$ ). In particular, the $\mathcal{Q}$-operator (7) used in the paper corresponds to indexes $a=(0, \downarrow)$ and $b=(1, \uparrow) \cdot \mathcal{B}_{0}^{\dagger}$ in Eq. (A3) is the $\mathbf{q}=0$ 'intra-sublevel' operator:

$$
\mathcal{B}_{\mathbf{q}}^{\dagger} \equiv \mathcal{N}_{\phi}^{-1 / 2} Q_{b b \mathbf{q}}^{\dagger}=\mathcal{N}_{\phi}^{-1} \sum_{p} \mathrm{e}^{-\mathrm{i} q_{x} p} b_{p+q_{y} / 2}^{\dagger} b_{p-q_{y} / 2}
$$

$\left(\mathcal{B}_{\mathrm{q}}=\mathcal{B}_{-\mathrm{q}}^{\dagger}\right)$. The $\mathcal{Q}$-operators form the closed Lie algebra with commutation rules

$$
\begin{aligned}
& {\left[Q_{c d \mathbf{q}_{1}}^{\dagger}, Q_{a b \mathbf{q}_{2}}^{\dagger}\right] \equiv \mathcal{N}_{\phi}^{-1 / 2}\left(\mathrm{e}^{-\mathrm{i} \mathbf{q}_{1} \times \mathbf{q}_{2} / 2} \delta_{b, c} Q_{a d \mathbf{q}_{1}+\mathbf{q}_{2}}^{\dagger}\right.} \\
& \left.-\mathrm{e}^{-i \mathbf{q}_{1} \times \mathbf{q}_{2} / 2} \delta_{a, d} Q_{c b \mathbf{q}_{1}+\mathbf{q}_{2}}^{\dagger}\right) .
\end{aligned}
$$

For a fixed pair of different indexes $(a, b)$ we have

$$
\left[\mathcal{Q}_{a b \mathbf{q}_{1}}^{\dagger}, \mathcal{Q}_{a b \mathbf{q}_{2}}^{\dagger}\right]=\left[\mathcal{Q}_{a b \mathbf{q}_{1}}, \mathcal{Q}_{a b \mathbf{q}_{2}}\right] \equiv 0
$$

where $a \neq b$, and

$$
\left[\mathcal{Q}_{a b \mathbf{q}_{1}}, \mathcal{Q}_{a b \mathbf{q}_{2}}^{\dagger}\right]=\mathrm{e}^{\mathrm{i} \mathbf{q}_{1} \times \mathbf{q}_{2} / 2} \mathcal{A}_{\mathbf{q}_{1}-\mathbf{q}_{2}}-\mathrm{e}^{\mathrm{i} \mathbf{q}_{2} \times \mathbf{q}_{1} / 2} \mathcal{B}_{\mathbf{q}_{1}-\mathbf{q}_{2}} .
$$

Besides,

$$
\begin{aligned}
& \mathrm{e}^{\mathrm{i} \mathbf{q}_{1} \times \mathbf{q}_{2} / 2}\left[\mathcal{A}_{\mathbf{q}_{1}}, \mathcal{Q}_{a b \mathbf{q}_{2}}^{\dagger}\right]=-\mathrm{e}^{-\mathrm{i} \mathbf{q}_{1} \times \mathbf{q}_{2} / 2}\left[\mathcal{B}_{\mathbf{q}_{1}}, \mathcal{Q}_{a b \mathbf{q}_{2}}^{\dagger}\right] \\
& =-\mathcal{N}_{\phi}^{-1} \mathcal{Q}_{a b q_{2}-\mathbf{q}_{1}}^{\dagger}
\end{aligned}
$$

and

$$
\begin{aligned}
& \mathrm{e}^{\mathrm{i} \mathbf{q}_{1} \times \mathbf{q}_{2} / 2}\left[\mathcal{A}_{\mathbf{q}_{1},} \mathcal{Q}_{a b \mathbf{q}_{2}}\right]=-\mathrm{e}^{-\mathrm{i} \mathbf{q}_{1} \times \mathbf{q}_{2} / 2}\left[\mathcal{B}_{\mathbf{q}_{1}}, \mathcal{Q}_{a b \mathbf{q}_{2}}\right] \\
& =\mathcal{N}_{\phi}^{-1} \mathcal{Q}_{a b q_{2}-\mathbf{q}_{1}} .
\end{aligned}
$$


In particular cases we get:

$$
\begin{aligned}
& {\left[\mathcal{Q}_{a b \mathbf{q}}, \mathcal{Q}_{a b \mathbf{q}}^{\dagger}\right]=\mathcal{A}_{0}-\mathcal{B}_{0} \text { and }} \\
& {\left[\mathcal{A}_{0}-\mathcal{B}_{0}, \mathcal{Q}_{a b \mathbf{q}}^{\dagger}\right]=-2 \mathcal{Q}_{a b \mathbf{q}}^{\dagger} / \mathcal{N}_{\phi} .}
\end{aligned}
$$

As applied to integer quantum Hall systems and in case we are interested only in first-order Coulomb corrections, only terms with $n_{a}+n_{c}=n_{b}+n_{d}$ not changing the cyclotron energy have to be kept in the general expression (A3) - these are terms commuting with the Hamiltonian of non-interacting electrons,

$$
\begin{aligned}
& \hat{H}^{(1)}-\frac{1}{2}\left|g \mu_{\mathrm{B}} B\right| S_{z}=\hbar \omega_{c} \mathcal{N}_{\phi} \sum_{a}\left(n_{a}+\frac{1}{2}\right) A_{0} \\
& -\frac{1}{2}\left|g \mu_{\mathrm{B}} B\right| \mathcal{N}_{\phi} \sum_{a} A_{0}\left(\delta_{\sigma_{a, \uparrow}}-\delta_{\sigma_{a, \downarrow}}\right)
\end{aligned}
$$

Besides, it is always possible to further reduce the number of terms taken into account in the Coulomb Hamiltonian, which occurs on specifying the type of excitation under study. Considerable simplifications appear in the study of single-exciton low-energy excitations in an integer quantum Hall system (see, for instance, the wellknown work [1]). The exciton mode is presented in the form $\mathcal{Q}_{a b q}|0\rangle$, where the $a$ sublevel is considered as fully occupied and the $b$ sublevel is completely empty (i.e. $\mathcal{A}_{\mathbf{q}}|0\rangle=\delta_{\mathbf{q}, 0}$ and $\mathcal{B}_{\mathbf{q}}|0\rangle=0$ ). The result is provided by the terms in Eq. (A3) non-commuting with $\mathcal{Q} t_{b q}$ and leading to states corresponding to the same changes of cyclotron numbers $\delta n=$ $n_{b}-n_{a}$ and spin numbers $\delta S_{z}=\sigma_{b}-\sigma_{a}$. Here we limit ourselves to the case where quantum numbers $\delta n$ and $\delta S_{z}$ uniquely determine the only possible mode corresponding to this type of excitation. In other words, the only pair $(a, b)$ is uniquely determined. This condition is met for the CSFE at the $v=2$ filling where $\delta n=\delta S_{z}=1$. Moreover, if $\delta n=\delta S_{z}=N>1$, we have the only realisation in the form of multi-excitonic states $|N\rangle$ or $|\tilde{N}\rangle$. However, there are more complicated situations, for example: (i) in the $v>1$ magnetoplasma modes, where $\delta n=1$ and $\delta S_{z}=0$ [1], two $(a, b)$ pairs are possible; (ii) in the $v=1$ CSFE, where $\delta n=-\delta S_{z}=1$ (see [17]), the single-exciton approach is not sufficient since the double-exciton mode with coupled magnetoplasma and spin-wave excitons becomes significant even in the first-order approximation. For the only pair $(a, b)$ the Coulomb Hamiltonian terms that have to be taken into account are as follows:

$$
\begin{aligned}
& \hat{H}_{\text {Coul }}^{\prime}=\hat{H}_{a}+\hat{H}_{b}+\hat{H}_{a b} \\
& +\sum_{c \neq a, b}\left(\hat{H}_{a c}+\hat{H}_{b c}\right) .
\end{aligned}
$$

Here

$$
\hat{H}_{a}=\sum_{q} \frac{\mathcal{F}_{e e}(q)}{q} h_{n_{a} n_{a} \mathbf{q}}^{2}\left(\mathcal{N}_{\phi} \mathcal{A}_{\mathbf{q}}^{\dagger} \mathcal{A}_{\mathbf{q}}-\mathcal{A}_{0}^{\dagger}\right)
$$

$\left[\hat{H}_{b}=\hat{H}_{a}(a \rightarrow b)\right]$ and

$$
\begin{aligned}
& \hat{H}_{d c}=\sum_{q} \frac{\mathcal{F}_{e e}(q)}{q}\left[h_{n_{d} n_{d} \mathbf{q}} h_{n_{c} n_{c} \mathbf{q}} \mathcal{N}_{\phi} \mathcal{D}_{\mathbf{q}}^{\dagger} C_{\mathbf{q}}\right. \\
& \left.+\left|h_{n_{d} n_{c} \mathbf{q}}\right|^{2} \delta_{\sigma_{d}, \sigma_{c}} Q_{d c \mathbf{q}}^{\dagger} Q_{d c \mathbf{q}}\right] .
\end{aligned}
$$

For CSFE at $v=2$ filling, where specifically $a=(0, \downarrow)$ and $b=(1, \uparrow)$, the relevant terms to study our excitation or even a magnetoexcitonic ensemble in the first-order approximation are

$$
\begin{aligned}
& \hat{H}_{\text {Coul }}^{\prime}=\sum_{q} \frac{\mathcal{F}_{e e}(q)}{2 q}\left[h_{00 \mathbf{q}}^{2}\left(\mathcal{N}_{\phi} \mathcal{A}_{\mathbf{q}}^{\dagger} \mathcal{A}_{\mathbf{q}}-\mathcal{A}_{0}^{\dagger}\right)\right. \\
& +h_{11 q}^{2}\left(\mathcal{N}_{\phi} \mathcal{B}_{\mathbf{q}}^{\dagger} \mathcal{B}_{\mathbf{q}}-\mathcal{B}_{0}^{\dagger}\right)+2 h_{00 \mathbf{q}} h_{11 \mathbf{q}} \mathcal{N}_{\phi} \mathcal{A}_{\mathbf{q}}^{\dagger} \mathcal{B}_{\mathbf{q}} \\
& \left.+2\left|h_{01 \mathbf{q}}\right|^{2} \mathcal{Q}_{b c \mathbf{q}}^{\dagger} \mathcal{Q}_{b c \mathbf{q}}\right],
\end{aligned}
$$

where $c$ represents the only sublevel: $c=(0, \uparrow)$.

Finally, we present results of the calculation of some mathematical expectations in the case of an integer filling factor. For the exciton state $\mathcal{Q}_{a b \mathrm{q}}^{\dagger}|0\rangle$, where the sublevel $a$ of the ground state is fully occupied, but $b$ is empty (i.e. $n_{a} \leq n_{b}$ ), we have

$$
\left\langle 0\left|\mathcal{Q}_{a^{\prime} b^{\prime} \mathbf{q}_{2}} \mathcal{Q}_{a b \mathbf{q}_{1}}^{\dagger}\right| 0\right\rangle=\delta_{a, a^{\prime}} \delta_{b, b^{\prime}} \delta_{\mathbf{q}_{1}, \mathbf{q}_{2}}
$$

The multi-exciton state

$$
|N, a b\rangle=\mathcal{Q}_{a b \mathbf{q}_{N}}^{\dagger} \mathcal{Q}_{a b \mathbf{q}_{N-1} \ldots}^{\dagger} \mathcal{Q}_{a b \mathbf{q}_{1}}^{\dagger}|0\rangle,
$$

when projected to another

$$
\left|N^{\prime}, a^{\prime} b^{\prime}\right\rangle=\mathcal{Q}_{a^{\prime} b^{\prime} \mathbf{q}_{N}^{\prime} N^{\prime}}^{\dagger} \mathcal{Q}_{a^{\prime} b^{\prime} \mathbf{q}_{N^{\prime}-1} \ldots}^{\dagger} \mathcal{Q}_{a^{\prime} b^{\prime} \mathbf{q}_{1}^{\prime}}^{\dagger}|0\rangle,
$$


leads to a non-zero result only if $N=N^{\prime}, a=a^{\prime}$, $b=b^{\prime}$ and $\mathbf{q}_{1}+\mathbf{q}_{2}+\ldots+\mathbf{q}_{N}=\mathbf{q}_{1}^{\prime}+\mathbf{q}_{2}^{\prime}+\ldots+\mathbf{q}_{N}^{\prime}$. In particular, the four- $\mathcal{Q}$ operator expectation value is

$$
\begin{aligned}
& \left\langle 0\left|Q_{a^{\prime} b^{\prime} \mathbf{q}_{2}^{\prime}} Q_{a^{\prime} b^{\prime} \mathbf{q}_{1}^{\prime}} Q_{a b \mathbf{q}_{1}}^{\dagger} Q_{a b \mathbf{q}_{2}}^{\dagger}\right| 0\right\rangle \\
& =\delta_{a, a^{\prime}} \delta_{b, b^{\prime}}\left(\delta_{\mathbf{q}_{2}^{\prime}, \mathbf{q}_{2}} \delta_{\mathbf{q}_{1}^{\prime}, \mathbf{q}_{1}}+\delta_{\mathbf{q}_{1}^{\prime}, \mathbf{q}_{2}} \delta_{\mathbf{q}_{2}, \mathbf{q}_{1}}\right. \\
& \left.-\frac{2 \cos \Phi}{N_{\phi}} \delta_{\mathbf{q}_{1}+\mathbf{q}_{2}, \mathbf{q}_{1}^{\prime}+\mathbf{q}_{2}^{\prime}}\right)
\end{aligned}
$$

where $\Phi=\left(\mathbf{q}_{1}{ }^{\prime} \times \mathbf{q}_{1}+\mathbf{q}_{2}{ }^{\prime} \times \mathbf{q}_{2}\right) / 2$.

Formula (29) for the squared norm $R_{N}$ can be proved for any state $|\tilde{N}, a b\rangle=\left(\mathcal{Q}_{a b q}^{\dagger}\right)^{N}|0\rangle$ by the technique of mathematical induction with the use of commutation rules (A4). When calculating the $P_{N \mathrm{p}}$ expectation (see Eq. (49)) by means of Eqs. (A4) we come to the recurrence relation

$$
\begin{aligned}
& P_{N \mathbf{p}}=2\left(\delta_{\mathbf{p}, 0}-\frac{1}{\mathcal{N}_{\phi}}\right) R_{N-1} \\
& +\frac{(N-2)\left(\mathcal{N}_{\phi}-N-1\right)}{\mathcal{N}_{\phi}} P_{N-1 \mathbf{p}},
\end{aligned}
$$

where $N \geq 2$ and $P_{2 \mathrm{p}}=2\left(\delta_{\mathrm{p}, 0}-1 / \mathcal{N}_{\phi}\right)$. The substitution $P_{N \mathbf{p}}=2\left(\delta_{\mathrm{p}, 0}-1 / \mathcal{N}_{\phi}\right)\left(1 / 2+\varepsilon_{N}\right) R_{N}$ results in a recursive relation for $\varepsilon_{N}$ which we write out just in the $N / \mathcal{N}_{\phi} \rightarrow 0$ limit: $\varepsilon_{N}=\varepsilon_{N-1}(1+2 / N)^{-1}$. The latter simple equation enables us to make the estimation $\varepsilon_{N}<O(1 / \ln N)$ if $N \gg 1$, hence, in the $1 \ll N \ll \mathcal{N}_{\phi}$ case we obtain formula (50).

\section{References}

[1] C. Kallin and B.I. Halperin, Excitations from a filled Landau level in the two-dimensional electron gas, Phys. Rev. B 30, 5655 (1984).

[2] S. Dickmann and I.V. Kukushkin, Zeromomentum cyclotron spin-flip mode in a spinunpolarized quantum Hall system, Phys. Rev. B 71, 241310(R) (2005).

[3] L.V. Kulik, I.V. Kukushkin, S. Dickmann, V.E. Kirpichev, A.B. Van'kov, A.L. Parakhonsky, J.H. Smet, K. von Klitzing, and W. Wegscheider, Cyclotron spin-flip mode as the lowest-energy excitation of unpolarized integer quantum Hall states, Phys. Rev. B 72, 073304 (2005).
[4] S. Dickmann, Relaxation of the cyclotron spinflip excitation in a spin-unpolarized quantum Hall system, Lith. J. Phys. 52, 96 (2012).

[5] S. Dickmann, Extremely slow spin relaxation in a spin-unpolarized quantum Hall system, Phys. Rev. Lett. 110, 166801 (2013).

[6] L.V. Kulik, A.V. Gorbunov, A.S. Zhuravlev, V.B. Timofeev, S. Dickmann, and I.V. Kukushkin, Super-long life time for 2D cyclotron spin-flip excitons, Sci. Rep. 5, 10354 (2015).

[7] A. Pinczuk, B.S. Dennis, D. Heiman, C. Kallin, L. Brey, C. Tejedor, S. Schmitt-Rink, L.N. Pfeiffer, and K.W. West, Spectroscopic measurement of large exchange enhancement of a spinpolarized 2D electron gas, Phys. Rev. Lett. 68, 3623 (1992).

[8] T. Ando, A.B. Fowler, and F. Stern, Electronic properties of two-dimensional systems, Rev. Mod. Phys. 54, 437 (1982).

[9] L.P.Gor'kov and I.E. Dzyaloshinskii, Contribution to the theory of the Mott exciton in a strong magnetic field, Sov. Phys. JETP 26, 449 (1968).

[10]A.B. Dzyubenko and Yu.E. Lozovik, Exact solutions and Bogoliubov transformations for zerodimensional electron-hole systems, Sov. Phys. Solid State 25, 874 (1983).

[11]A.B. Dzyubenko and Yu.E. Lozovik, Quasi-2D electron-hole pair condensate in strong magnetic fields, Sov. Phys. Solid State 26, 938 (1984).

[12]A.S. Zhuravlev, S. Dickmann, L.V. Kulik, and I.V. Kukushkin, Slow spin relaxation in a quantum Hall ferromagnet state, Phys. Rev. B 89, 161301(R) (2014).

[13]I.V. Lerner and Yu.E. Lozovik, Mott exciton in a quasi-two-dimensional semiconductor in a strong magnetic field, Sov. Phys. JETP 51, 588 (1980).

[14]S.V. Iordanski and Y. Levinson, Electron-phonon scattering in a two-dimensional electron gas in a very strong magnetic field near the percolation threshold, Phys. Rev. B 53, 7308 (1996).

[15]A.B. Dzyubenko and Yu.E. Lozovik, Symmetry of Hamiltonians of quantum two-component systems: condensate of composite particles as an exact eigenstate, J. Phys. A 24, 415 (1991). 
[16]R.H. Dicke, Coherence in spontaneous radiation processes, Phys. Rev. 93, 99 (1954).

[17]S. Dickmann and V.M. Zhilin, Double-exciton component of the cyclotron spin-flip mode in a quantum Hall ferromagnet, Phys. Rev. B 78, 115302 (2008).

KOHERENTINIUU IR NEKOHERENTINIỤ SUKINIO SUŽADINIMU ANSAMBLIỤ NEPOLIARIZUOTOJE KVANTINĖJE HOLO SISTEMOJE OPTINĖ SUGERTIS

\author{
S. Dickmann
}

Rusijos mokslų akademijos Kietojo kūno fizikos institutas, Černogolovka, Rusija 